\title{
Neural substrates of cognitive flexibility in cocaine and gambling addictions
}

Antonio Verdejo-Garcia, Luke Clark, Juan Verdejo-Román, Natalia Albein-Urios, José M. Martinez-Gonzalez, Blanca Gutierrez and Carles Soriano-Mas

\section{Background}

Individuals with cocaine and gambling addictions exhibit cognitive flexibility deficits that may underlie persistence of harmful behaviours.

\begin{abstract}
Aims
We investigated the neural substrates of cognitive inflexibility in cocaine users $v$. pathological gamblers, aiming to disambiguate common mechanisms $v$. cocaine effects.

\section{Method}

Eighteen cocaine users, 18 pathological gamblers and 18 controls performed a probabilistic reversal learning task during functional magnetic resonance imaging, and were genotyped for the DRD2/ANKK Taq1A polymorphism.
\end{abstract}

\section{Results}

Cocaine users and pathological gamblers exhibited reduced ventrolateral prefrontal cortex (PFC) signal during reversal shifting. Cocaine users further showed increased dorsomedial PFC (dmPFC) activation relative to pathological gamblers during perseveration, and decreased dorsolateral
PFC activation relative to pathological gamblers and controls during shifting. Preliminary genetic findings indicated that cocaine users carrying the DRD2/ANKK Taq1A1+ genotype may derive unique stimulatory effects on shifting-related ventrolateral PFC signal.

\section{Conclusions}

Reduced ventrolateral PFC activation during shifting may constitute a common neural marker across gambling and cocaine addictions. Additional cocaine-related effects relate to a wider pattern of task-related dysregulation, reflected in signal abnormalities in dorsolateral and dmPFC.

\section{Declaration of interest}

L.C.: The Centre for Gambling Research at UBC is funded by support from the Province of British Columbia and the British Columbia Lottery Corporation. The other authors declare no conflicts of interest concerning this study.

\section{Copyright and usage}

(c) The Royal College of Psychiatrists 2015.
Cocaine dependence and pathological gambling (relabelled gambling disorder ${ }^{1}$ ) have recently been jointly classified as addictive disorders, based on evidence for overlap in terms of clinical presentation, neurobiological profile and genetic liability. ${ }^{1}$ In treatment-seeking samples, both disorders are characterised by persistent engagement with the harmful behaviour despite its adverse consequences in interpersonal and occupational domains. This persistence reflects cognitive inflexibility in updating and integrating the values of potential actions with reference to previous and expected outcomes. ${ }^{2}$ In addition to its relevance to theories of addiction, cognitive inflexibility is also clinically relevant because perseverative responding is a significant predictor of poorer addiction treatment outcomes. ${ }^{3}$ Persistent responding in the face of negative feedback can be modelled experimentally using the reversal learning paradigm, a discrimination task in which participants learn to respond to a reinforced stimulus, but must then learn to respond to another, previously irrelevant, stimulus. ${ }^{4}$ Two complementary aspects of reversal learning are relevant to addictive behaviours: the strong motivational tendency to respond to previously reinforced stimuli and a difficulty in flexibly shifting towards novel reinforcing stimuli, which are collectively indicated by 'perseveration. ${ }^{5}$

Behavioural studies indicate that both cocaine users and pathological gamblers exhibit reversal learning perseveration compared with controls. ${ }^{6}$ In cocaine users, perseveration is mainly observed following changes in response-outcome contingencies (i.e. in reversal learning tasks involving probabilistic wins and losses). ${ }^{7,8}$ By contrast, flexible responding is relatively preserved in non-rewarded stimulus-discrimination tasks involving fixed schedules. ${ }^{9}$ In pathological gamblers, there is reduced flexibility after reversal of previously rewarded contingencies. ${ }^{10}$ Reversal learning is underpinned by neural circuitry comprising dorsomedial, dorsolateral and ventrolateral prefrontal cortex (dmPFC, dlPFC and vlPFC respectively) and their connections with the striatum and amygdala. ${ }^{11}$ Accordingly, reversal learning perseveration has been associated with abnormal dmPFC and dlPFC function in cocaine users ${ }^{12,13}$ and decreased activation of right vlPFC in pathological gamblers. ${ }^{14}$ Moreover, there is evidence that reversal learning is genetically mediated by dopamine D2 expression, as healthy volunteers carrying the DRD2/ANKK1 Taq1A A1 allele (linked to decreased D2 receptor availability) exhibit poorer behavioural performance, and blunted vlPFC activation during shifting. ${ }^{15}$ The A1 allele has also been associated with cocaine and gambling addictions. ${ }^{16}$

Accepting the substantial neurobiological overlap between these disorders, cocaine addiction nevertheless involves significant drug-induced, active, detrimental effects on the dopaminergic system. ${ }^{17-19}$ Preclinical studies have demonstrated that prolonged cocaine administration induces significant reversal learning deficits, ${ }^{20}$ and that these changes are mediated by D2-dopamine transmission. ${ }^{21}$ In humans, comparable effects are indicated by correlations against cocaine chronicity in neuropsychological studies ${ }^{22,23}$ but there is no evidence regarding the specificity of these deficits with respect to the behavioural addictions. We reasoned that cocaine-induced neuroadaptive changes should be absent in pathological gamblers. ${ }^{24}$ As such, pathological gambling can serve as a control group to disambiguate the active detrimental effects of cocaine dependence on reversal learning while accounting for shared vulnerability and reinforcement history. ${ }^{25,26}$ The primary aim of this study was to contrast brain activation patterns associated with cognitive shifting and response perseveration in individuals diagnosed with cocaine dependence $v$. 
pathological gambling. We hypothesised that cocaine dependence, compared to a behavioural addiction, would be associated with distinctive alterations in prefrontal regions recruited by reversal learning. As a secondary exploratory aim, we sought to examine whether this brain dysregulation was dopaminergically linked, by studying associations with the DRD2/ANKK1 Taq1A genetic variant.

\section{Method}

\section{Participants}

The sample consisted of 54 participants: 18 individuals meeting DSM-IV-TR criteria for cocaine dependence (cocaine users) not meeting criteria for any other Axis I or Axis II disorder, 18 individuals meeting DSM-IV-TR criteria for pathological gambling (gamblers) not meeting criteria for any other Axis I or Axis II disorder, and 18 healthy comparison individuals who did not meet DSM-IV-TR criteria for Axis I or Axis II disorders (controls). Table 1 presents sociodemographic information. The three groups did not differ significantly in age, years of education, or IQ measured by the Kaufman Brief Intelligence Test. ${ }^{27}$ Table 1 also presents drug/gambling use characteristics (monthly amount and duration of use) as recorded by the Interview for Research on Addictive Behaviors ${ }^{28}$ and psychological symptoms as measured by the General Health Questionnaire. ${ }^{29}$ Participants had very limited exposure to drugs other than cocaine, alcohol or tobacco; less than $20 \%$ of participants had used cannabis, approximately $5 \%$ had used MDMA or hallucinogens, and no participants had used amphetamines or opiates. Table 1 also displays data on self-reported abstinence duration. In cocaine users the mean duration of abstinence was 2.7 months and in gamblers the mean duration of abstinence was 5.7 months. In addition, abstinence was monitored for 3 weeks during the study, measured with urine tests for alcohol and drug use and cross-checked self- and collateral reports for gambling. Table 1 also displays DRD2/ANKK Taq1A genotype distributions. In agreement with population-based data, ${ }^{30,31}$ the $\mathrm{A} 1+$ genotype was found in between 30 and $40 \%$ of participants within each group. Sociodemographic, drug/gambling use and psychological characteristics by genotype subgroups are presented in online Table DS1.

Online Fig. DS1 displays a flowchart of the recruitment process. Cocaine users were recruited as they commenced treatment in the outpatient clinic Centro Provincial de Drogodependencias in Granada (Spain). Gamblers were recruited as they commenced treatment in the outpatient clinic Asociación Granadina de Jugadores en Rehabilitación in Granada (Spain). Both clinics provide psychological therapies for addictive disorders. The inclusion criteria were as follows: (a) aged between 18 and 45 years; (b) estimated IQ levels above 80; (c) meeting DSM-IV-TR criteria for cocaine dependence or pathological gambling - as assessed by the Structured Clinical Interview for

\section{Table 1 Demographic and clinical characteristics of the three study groups}

\begin{tabular}{|c|c|c|c|c|}
\hline Demographic variables & $\begin{array}{l}\text { Controls } n=18 \\
\text { Mean (s.d.) }\end{array}$ & $\begin{array}{l}\text { Gamblers } n=18 \\
\text { Mean (s.d.) }\end{array}$ & $\begin{array}{c}\text { Cocaine users } n=18 \\
\text { Mean (s.d.) }\end{array}$ & $P$ \\
\hline Age (years) & $31.17(4.74)$ & $33.56(7.97)$ & $34.27(6.87)$ & 0.349 \\
\hline Gender (male/female) & $17 / 1$ & $16 / 2$ & $17 / 1$ & 0.774 \\
\hline Laterality (right-left) & $17 / 1$ & $17 / 1$ & $14 / 4$ & 0.193 \\
\hline Years of education & $10.56(1.92)$ & $10.28(2.11)$ & $9.78(1.66)$ & 0.468 \\
\hline Verbal IQ & $106.89(8.98)$ & $102.67(7.39)$ & $100.94(7.58)$ & 0.082 \\
\hline \multicolumn{5}{|l|}{ DRD2/ANKK Taq1A1+genotype } \\
\hline $\mathrm{A} 1+$ & 6 & 7 & 5 & \\
\hline A1- & 12 & 10 & 13 & \\
\hline \multicolumn{5}{|l|}{ Clinical variables } \\
\hline \multicolumn{5}{|l|}{ Cocaine (0 HC/ 0 PG/ 18 CDI) } \\
\hline Age at onset cocaine use (years) & & & $21.28(5.83)$ & \\
\hline Monthly amount cocaine users (g) & & & $16.86(25.49)$ & \\
\hline Duration cocaine (months) & & & $43.75(36.32)$ & \\
\hline Abstinence cocaine (months) & & & $2.73(5.43)$ & \\
\hline \multicolumn{5}{|l|}{ Gambling (0 HC/ 18 PG/ 0 CDI) } \\
\hline Age at onset gambling (years) & & $22.17(8.71)$ & & \\
\hline Monthly amount gambling (h) & & $42.53(41.47)$ & & \\
\hline Duration gambling (months) & & $26.12(24.56)$ & & \\
\hline Abstinence gambling (months) & & $7.69(6.51)$ & & \\
\hline \multicolumn{5}{|l|}{ Tobacco (8 HC/ 8 PG/ 14 CDI) } \\
\hline Age at onset tobacco use (years) & $17.75(5.55)$ & $15.50(3.51)$ & $15.71(2.58)$ & 0.411 \\
\hline Monthly tobacco use (cig) & $286.25(222.90)$ & $667.50(278.55)$ & $564.29(362.21)$ & 0.051 \\
\hline Duration tobacco (months) & $76.37(104.25)$ & $175.50(101.00)$ & $137.57(121.16)$ & 0.219 \\
\hline \multicolumn{5}{|l|}{ Alcohol (7 HC/ 14 PG/ 15 CDI) } \\
\hline Age at onset alcohol use (years) & $19.14(5.53)$ & $16.29(1.70)$ & $17.87(4.55)$ & 0.411 \\
\hline Monthly alcohol use (SDU) & $10.07(9.75)$ & $17.43(20.09)$ & $31.69(20.70)$ & 0.038 \\
\hline Duration alcohol (months) & $83.75(56.21)$ & $75.43(63.31)$ & $88.69(93.90)$ & 0.928 \\
\hline \multicolumn{5}{|l|}{ Cannabis (4 HC/ 1 PG/ 6 CDI) } \\
\hline Age at onset alcohol use (years) & $18.75(3.77)$ & 25 & $18.71(7.47)$ & 0.664 \\
\hline Monthly cannabis use (joints) & $0.96(0.75)$ & 4 & $107.83(125.57)$ & 0.267 \\
\hline Duration cannabis (months) & $19.25(19.35)$ & 4 & $110(137.33)$ & 0.405 \\
\hline GHQ somatic symptoms & $0.39(0.85)$ & $1.72(2.33)$ & $1.43(1.86)$ & 0.079 \\
\hline GHQ anxiety & $1.28(2.19)$ & $1.64(2.34)$ & $2.12(2.50)$ & 0.577 \\
\hline GHQ social dysfunction & $0.83(1.65)$ & $1.00(1.84)$ & $1.25(1.91)$ & 0.796 \\
\hline GHQ depression & $0.44(0.98)$ & $1.27(2.15)$ & $1.50(2.42)$ & 0.248 \\
\hline
\end{tabular}


DSM-IV Disorders - Clinician Version (SCID-I-CV); ${ }^{32}$ (d) being treatment commencers; and (e) abstinence duration $>15$ days. Abstinence in the cocaine users was confirmed by two urine tests per week plus an ad hoc test on the scanning day itself. Positive urine toxicologies for any other drug were also exclusionary. Gambling abstinence in the pathological gamblers was assessed by self-report cross- validated by spouses or relatives. The exclusion criteria were: (a) presence of any other Axis I or Axis II disorders, with the exceptions of alcohol misuse and nicotine dependence; (b) history of head injury or neurological, infectious, systemic or any other diseases affecting the central nervous system; (c) having followed other treatments within the 2 years preceding study onset and (d) having entered treatment by court request. Comorbid Axis I disorders were assessed with the SCID-I-CV. Axis II disorders were assessed using the International Personality Disorders Examination (IPDE). ${ }^{33}$ We also used the Conners' Adult ADHD Diagnostic Interview for DSM-IV (CAADID) ${ }^{34}$ to assess adult ADHD (which was also exclusionary). Controls were recruited from local employment agencies. In addition to the former exclusion criteria, healthy controls could not meet any diagnosis of substance use disorders - with the exception of nicotine dependence. Axis I and II disorders were also assessed in this group using the SCID-I-CV, the IPDE and the CAADID. All the diagnoses were made by a registered clinical psychologist.

The study was approved by the Ethics Committee for Research in Humans of the University of Granada (Spain). All participants signed an informed consent form certifying their voluntary participation

\section{Functional MRI (fMRI) task}

We used the probabilistic reversal learning task, as described in Cools et al. ${ }^{11}$ In each trial, participants were required to choose between two stimuli (abstract, coloured patterns) presented to the left and right visual fields (location was randomised). Participants were told that, according to a predefined rule, one stimulus was correct on each trial (the CS+), and the other stimulus (the $\mathrm{CS}-$ ) was incorrect. At various points throughout the task, the rule deciding the correct stimulus would change; the participant should change their response when they were confident that the rule had changed. The task employed probabilistic feedback such that the CS+ was rewarded $\sim 85 \%$ of times, and the CS - was rewarded $\sim 15 \%$ of times. This gave rise to two types of errors: probabilistic errors (where participants chose the correct stimulus but received negative feedback), and perseverative errors (where participants keep responding to the previously reinforced stimuli, despite negative feedback). The task was trained before scanning (using slightly different stimuli) and then implemented inside the scanner in 3 consecutive blocks of $11 \mathrm{~min}$ each. Each block consisted of 10 discrimination stages, yielding 9 reversals. Reversal of the stimulus-reward contingency occurred after 10 to 15 correct responses (including probabilistic errors). The number of probabilistic errors between each reversal varied from 0 to 4 .

Stimuli were presented through magnetic-resonance-compatible liquid-crystal display goggles (Resonance Technology, Northridge, CA, USA). Behavioural responses were recorded through a fivebutton box, Evoke Response Pad System (Resonance Technology Inc.). On each trial, stimuli were presented for $2000 \mathrm{~ms}$, within which time the response had to be made (or else a 'too late' message was presented). Participants responded using the left or right button on a button box positioned on participants' chest. Feedback was a green 'smiley' face for correct responses, and a red sad face for incorrect responses, and was presented immediately after the participants' response. The feedback faces were presented centrally for $500 \mathrm{~ms}$, during which time the stimuli also remained on the screen. Following feedback, there was a variable inter-trial interval (a fixation cross) that was adjusted so that the overall interstimulus interval was $3253 \mathrm{~ms}$, enabling precise desynchronisation from the repetition time (TR) (of $2000 \mathrm{~ms}$ ) and sufficient sampling across the hemodynamic response function.

\section{Behavioural measures}

The main performance measures were hit rates (proportion of correct responses by total trials), number of perseverative errors, and perseveration error rates (number of perseverative errors divided by number of sequences on which the perseveration criterion was met). To meet the perseveration criterion, participants had to make at least one consecutive response to the previously rewarded stimulus immediately following reversal.

\section{Imaging data acquisition and preprocessing}

We used a 3.0 Tesla clinical MRI scanner, equipped with an eightchannel phased-array head coil (Intera Achieva, Philips Medical Systems, Eindhoven, The Netherlands). During acquisition, three T2*-weighted echo-planar imaging (EPI) was obtained $(\mathrm{TR}=2000 \mathrm{~ms}$, echo time $(\mathrm{TE})=35 \mathrm{~ms}$, field of view $(\mathrm{FOV})=230 \times 230 \mathrm{~mm}, 96 \times 96$ matrix, flip angle $=90^{\circ}, 214-\mathrm{mm}$ axial slices, 1-mm gap, 330 scans each). A sagittal three-dimensional T1-weighted turbo-gradient-echo sequence ( 160 slices, TR $=8.3 \mathrm{~ms}$, $\mathrm{TE}=3.8 \mathrm{~ms}$, flip angle $=8^{\circ}, \mathrm{FOV}=240 \times 240,1 \mathrm{~mm}^{3}$ voxels) was obtained in the same experimental session for anatomical localisation of functional findings.

The brain images were analyzed using Statistical Parametric Mapping software (Wellcome Department of Cognitive Neurology, Institute of Neurology, Queen Square, London, UK), running under Matlab R2009 (MathWorks, Natick, MA, USA). Preprocessing steps were slice timing correction, re-slicing to the first image of the time series, normalisation (using affine and smoothly non-linear transformations) to an EPI template in the Montreal Neurological Institute space, and spatial smoothing by convolution with a $3 \mathrm{D}$ Gaussian kernel (full width at half maximum $(\mathrm{FWHM})=8 \mathrm{~mm}$ ).

\section{DRD2/ANKK1 Taq1A genotyping}

The DRD2/ANKK Taq1A polymorphism (rs1800497) is located in a putative substrate binding domain of the ANNK1 gene and results in a Glu713Lys substitution. In our study, this polymorphism was characterised using a TaqMan allelic discrimination assay from Life Technologies. Cycling was performed on a StepOne Plus thermocycler with conditions recommended by Life Technologies.

Three genotypes of the dopamine DRD2/ANNK1-TaqIa locus can be differentiated: the A1A1 genotype, the A1A2 genotype, and the A2A2 genotype. Because of the small prevalence of the A1A1 genotype (3\% of the healthy White population), A1A1 and A1A2 participants are commonly grouped as $\mathrm{A} 1+$ participants, whereas A2A2 participants are referred to as A1- participants. The prevalence of at least one A1 allele (A1+ group) has been associated with an up to $30 \%$ reduction in D2 receptor density. ${ }^{35}$

\section{Statistical analyses}

\section{Behavioural analyses}

Behavioural data were analysed with SPSS version 19. We conducted one-way ANOVAs followed by Tukey tests to compare the three groups on the reversal learning behavioural measures. We also conducted two-way ANOVAs (with Group and DRD2/ ANKK Taq1A genotype as factors) to examine differences between genotype subgroups. 


\section{Neuroimaging analyses}

The time series were high-pass filtered (128s), and a canonical hemodynamic response function was modeled to a delta function at participants' response on each trial, which co-occurred with the presentation of the feedback. The following events were modeled: (a) correct responses; (b) perseverative errors (errors following a rule change where participants fail to switch response); (c) final reversal errors (last negative feedback in the series of perseverative errors followed by a response switch); and (d) probabilistic errors (correct responses for which misleading negative feedback was given). Error trials that could not be classified as probabilistic or reversal errors were not included in the model. The main contrast of interest was final reversal errors $v$. perseverative errors, which reflects the behavioural shift component. We also calculated the correct $v$. incorrect (and the reversed incorrect $v$. correct) contrast to map positive and negative feedback-related activation, and perseverative minus probabilistic errors to map persistent responding controlling for negative feedback.

One-sample $t$-tests were conducted on the resulting first-level contrast images to assess within-group activations in each of the contrasts. These results were corrected for multiple comparisons with a combination of voxel intensity and cluster extent thresholds. The spatial extent threshold was determined by 1000 Monte Carlo simulations using AlphaSim, ${ }^{36}$ as implemented in the SPM REST toolbox. ${ }^{37}$ The input parameters included brain mask of 152295 voxels, an individual voxel threshold probability of 0.005 and a cluster connection radius of $5 \mathrm{~mm}$, at 10.2, 10.4 and 9.1 FWHM smoothness for the contrasts correct $v$. incorrect, final reversal errors $v$. perseverative errors and perseverative $v$. probabilistic errors, respectively. A minimum cluster extent of 262, 260 and 212 voxels respectively was estimated to satisfy a family-wise error (FWE) corrected $P$-value of $P_{\text {FWE }}<0.05$. Next, we conducted a series of three group ANOVAs to assess between-group differences using the same first-level contrast images. Statistical significance in these tests was defined by the same input parameters, masking results by the activation maps derived from the one-sample $t$-tests. Therefore, for the contrasts correct $v$. incorrect (and incorrect $v$. correct), final reversal errors $v$. perseverative errors and perseverative minus probabilistic errors, respectively, a minimum cluster extent of 104, 14, 47 and 12 voxels (within brain masks of 41 129, 1812, 14149 and 1009 voxels), was estimated to satisfy a $P_{\mathrm{FWE}}<0.05$. In those contrasts yielding significant group differences, we conducted additional analyses in SPSS to assess Group $\times$ DRD2/ANKK Taq1A Genotype interactions on brain activation clusters differing between groups. Specifically, we conducted two-way ANOVAs (with Group and DRD2/ANKK Taq1A genotype as factors) on peak activations derived from the fMRI contrasts, followed by relevant pairwise comparisons. To exclude a potential performance confound, all analyses were replicated controlling for the behavioural measures of final reversal errors and perseverative errors. Results were equivalent in both approaches, and hence we only report the original, non-covaried analyses. Likewise, since both tobacco and alcohol use have been linked to reversal performance and dopaminergic function, we conducted additional analyses including the monthly amount and duration of use of these substances as covariates. Further, we conducted a series of twoway ANOVAs to examine whether smoking use or cannabis use status interacted with Group or DRD2/ANKK Taq1A genotype effects on peak activations derived from the fMRI contrasts.

\section{Correlation analyses}

Correlation analyses were performed in SPSS using the peak activations derived from the fMRI contrasts. The beta eigenvalues corresponding to each region were extracted for each participant, and then correlated with the behavioural measures of number of perseverative errors and perseveration error rates. We also correlated the beta eigenvalues corresponding to each region with self-report estimates of abstinence duration.

\section{Results}

\section{Behavioural measures}

Behavioural measures are presented in Table 2. The three groups only differed in the rate of perseverative errors, with cocaine users committing more perseverative errors than pathological gamblers or controls. In addition, in the cocaine group, perseveration errors were correlated positively with lifetime duration of cocaine use $(r=0.470, P=0.025)$. By contrast, perseveration error rates were negatively correlated with time since gambling onset in the pathological gamblers $(r=-0.409$, $P=0.049$ ). Group $\mathrm{x} D R D 2 / A N K K$ Taq1A genotype analyses showed no significant interaction effects on behavioural measures.

\section{Neuroimaging}

\section{Correct $v$. incorrect responses}

Collapsing across groups, correct ( $v$. incorrect) responses were associated with increased signal in striatum, superior and medial frontal gyri, lateral orbitofrontal cortex, anterior and posterior cingulate, posterior insula, amygdala, superior temporal gyri, angular gyri and occipital regions. The reverse contrast indicated signal associated with negative feedback in right dlPFC, right insula and supplementary motor area (see online Table DS2 and Fig. DS2). Between-group comparisons indicated no reliable differences in these contrasts.

\section{Final reversal errors $v$. perseverative errors}

Shifting (i.e. final reversal errors) was associated with significant signal increases across all groups in dorsal anterior cingulate cortex, bilateral anterior insular/orbitofrontal cortex, right dorsolateral prefrontal and ventrolateral prefrontal cortices, inferior

\begin{tabular}{|c|c|c|c|c|}
\hline & $\begin{array}{c}\text { Controls } \\
\text { Mean (s.d.) }\end{array}$ & $\begin{array}{l}\text { Gamblers } \\
\text { Mean (s.d.) }\end{array}$ & $\begin{array}{l}\text { Cocaine users } \\
\text { Mean (s.d.) }\end{array}$ & $P$ \\
\hline Hit rate (\% correct responses) & $64.25(7.29)$ & $63.39(8.32)$ & $62.02(8.53)$ & 0.705 \\
\hline Perseverative errors & $21.72(9.38)$ & $20.94(8.53)$ & $27.61(12.79)$ & 0.120 \\
\hline Sequences on which criterion for perseveration was met & $14.94(6.71)$ & $14.72(6.72)$ & $15.56(6.56)$ & 0.927 \\
\hline Perseveration error rate & $1.49(0.26)$ & $1.48(0.40)$ & $1.87(0.75)$ & 0.039 \\
\hline Total trials to complete the task & $508.50(70.45)$ & $517.50(77.66)$ & $530.17(85.64)$ & 0.707 \\
\hline
\end{tabular}


parietal cortex, striatum, thalamus and posterior visual areas extending to the fusiform gyrus. Final reversal errors were also associated with reduced signal in the rostral anterior cingulate and medial frontal gyri, the posterior cingulate gyrus and the left angular and parahippocampal gyri (online Fig. DS3 and Table DS3).

Pairwise between-group comparisons showed that both cocaine users and pathological gamblers had significantly decreased activation in the right vlPFC (inferior frontal gyrus) compared to controls. In addition, the cocaine users had significantly decreased activation in the right dlPFC (middle frontal gyrus) compared with both pathological gamblers and controls (online Fig. DS3 and Table DS3). We found no significant correlations with behavioural measures.

\section{Perseverative $v$. probabilistic errors}

Perseveration was associated with significant activation in superior and medial frontal gyri and rostral anterior cingulate gyrus. Between-group comparisons showed no significant differences between cocaine users or gamblers compared to controls, although the cocaine users did display significantly higher activation than pathological gamblers in the medial frontal gyrus (see online Fig. DS4). Medial frontal gyrus activation was also negatively correlated with the number of perseverative errors in cocaine users ( $r=-0.470, P=0.025$ ), but positively correlated with the number of perseverative errors in pathological gamblers $(r=0.467$, $P=0.025$, see online Fig. DS4).

\section{Group $\times$ DRD2/ANKK Taq1A genotype interactions}

Clusters showing significant between-group differences were further examined in relation to Group $\times$ DRD2/ANKK Taq1A genotype analyses. There was a significant group $\times$ genotype interaction $(F(2,46)=4.81, P=0.013)$ in the right vlPFC, driven by opposing effects of the dopamine genotype in the cocaine users relative to the other two groups (see online Fig. DS5). Pairwise analyses showed that within A1- carriers, cocaine users had lower activation than both pathological gamblers and controls. Conversely, cocaine A1+ carriers had significantly higher activation than pathological gamblers of the same genotype. No further significant interactions were observed.

\section{Correlation between patterns of brain activation and abstinence duration}

We did not find significant correlations between task-related brain activations and duration of abstinence of cocaine or gambling use.

\section{Sensitivity analyses}

Covariate models including monthly amount and duration of alcohol and tobacco use did not change the overall pattern of results. Further, smoking status (smokers $v$. non-smokers) and cannabis use status (cannabis users $v$. non-users) showed no significant interactions with Group or Genotype on any of the peak activations derived from fMRI analyses.

\section{Discussion}

Our results demonstrate that reduced signal in right vlPFC during shifting is common to both cocaine users and pathological gamblers. This shared effect was supplemented by a wider pattern of task-related dysregulation in the cocaine users, with decreased right dlPFC activation during shifting, and increased medial prefrontal cortex activation during perseveration. These brain activation differences were paralleled by behavioural results, where the cocaine users committed more perseverative errors compared with both pathological gamblers and controls. Preliminary exploratory genetic analyses of the DRD2/ANKK Taq1A genotype suggest an underlying dopaminergic contribution to reversalrelated brain activity: both controls and pathological gamblers carrying the (high risk) A1+ genotype had decreased switchrelated vlPFC signal, but this pattern was reversed in the cocaine group, in which the A1+ carriers exhibited greater shifting-related activation.

Our task activation results replicate the well-described pattern of dorsal prefrontal/insular activations in response to error-related negative feedback, in concert with recruitment of more ventral and lateral aspects of prefrontal cortex, anterior cingulate and striatum during shifting. ${ }^{11,38}$ Both cocaine users and pathological gamblers showed diminished activation of the right vlPFC during shifting, consistent with the pattern previously described in pathological gamblers performing a similar reversal task. ${ }^{14}$ The right vlPFC is a key region for successful self-control of behaviour and emotional regulation. ${ }^{39}$ Further, previous fMRI studies have shown that right vlPFC activation is distinctively increased in individuals with high resilience to addiction, ${ }^{40}$ and decreased in individuals with family risk of addiction. ${ }^{41}$ We infer that dysfunction of this region is commonly involved in both cocaine and gambling addictions. The increased shifting-related activation in cocaine users carrying the (high risk) Al allele compared to pathological gamblers carrying the same allele might be explained by the dopamine inverted-U principle, by which Al+ carriers, with lower dispositional dopamine function, may derive stimulatory 'benefits' from cocaine-induced changes. ${ }^{42}$ For example, treatment with the dopamine D2-receptor agonist cabergoline in healthy volunteers provoked opposite effects in $\mathrm{A} 1-v$. A1+ carriers, also manifested in the activation of the right vlPFC. ${ }^{43}$ However, because of the small sample size of genotype subgroups, these findings should be treated as preliminary, and could alternatively be explained by linked involvement of other dopamine gene polymorphisms. For example, recent evidence suggests that the dopamine agonist tolcapone has unique stimulatory effects on prefrontal cortex activity in smokers carrying the COMT val/val genotype, which is also associated with lower dopamine function. ${ }^{44}$ Future, adequately powered, molecular genetic studies are warranted to test the notion of whether individuals with low dispositional dopamine transmission derive stimulatory brain effects from cocaine consumption.

In addition to overlapping deficits, cocaine users showed decreased right dlPFC activation (BA 9) compared with gamblers and controls during shifting, and increased medial frontal gyrus activation (BA 10) compared with gamblers during perseveration. BA 9 has been specifically involved in the updating of the working memory stores that set stimulus-response contingencies, ${ }^{45}$ whereas BA10 has been primarily involved in the control of stimulus-oriented attention. ${ }^{46}$ Therefore, both findings are compatible with the model-based $v$. model-free systems approach to reversal learning. ${ }^{2}$ According to this model, cocaine-induced changes affect systems involved in the updating of stimulusoutcome values that serve to adjust predictions about future outcomes. Preclinical studies have shown cocaine-induced neural adaptations in the rat prelimbic cortex (the functional homologue of the human dlPFC), ${ }^{47,48}$ which is critical for the learning of novel stimulus-outcome associations. ${ }^{49}$ Similarly, in humans, duration of cocaine use is negatively associated with BA 9 gray matter volumes. ${ }^{50}$ As a consequence of neuroadaptations in brain regions involved on model-based predictions, cocaine users may become more dependent on systems handling model-free cached 
representations of stimulus-outcome values. ${ }^{2}$ We propose that this compensatory mechanism would be exemplified by significantly increased medial prefrontal activation during perseveration in cocaine users compared to gamblers. Therefore, our results suggest that cocaine users require additional recruitment of medial prefrontal regions to compensate for compromised lateral prefrontal regions specialised in the updating of stimulus-outcome predictions. Conversely, pathological gamblers engage this region to a lesser extent than both cocaine users and controls, and this pattern correlates with fewer perseverations within this group (see Dombrovski et $a l^{51}$ for a similar effect in depression, which might be explained by these populations being overly sensitive to misleading probabilistic feedback, hence less likely to perseverate in this task). This interpretation is also consistent with the neuropsychological profile we have described previously for this cohort, where the cocaine users have a selective impairment in working memory compared to the pathological gamblers (i.e. poorer updating of stimulus-outcome values), whereas the pathological gamblers have steeper delay discounting (i.e. enhanced reinforcement sensitivity). ${ }^{52}$

Our results illustrate the neural underpinnings of reversal learning in cocaine and gambling addictions. Since reversal learning is a well-validated translational model of inflexibility/ perseveration, and is linked to addiction severity and clinical prognosis, these results inform both mechanistic and clinical research in addictive disorders. In regards to clinical implications, our findings suggest that brain stimulation and/or cognitive enhancement interventions targeting the dlPFC may contribute to the alleviation of perseveration in the context of cocaine addiction. ${ }^{53}$ We note that our fMRI procedure did not detect significant behavioural alterations in reversal learning performance between gamblers and controls. One possible inference is that signs of overt compulsivity may be less in pathological gamblers, compared with cocaine addiction. However, the probabilistic task involved serial reversals on a semi-regular schedule in order to optimise case-by-case recruitment of fronto-striatal neural circuitry, but with reduced behavioural sensitivity to group differences. The observation that gamblers showed a differential pattern of reversal-related brain activity is compatible with the neuropsychological differences found in previous studies using behaviour-sensitive reversal tasks. ${ }^{10}$ In this regard, our findings suggest that brain stimulation and/or cognitive enhancement interventions targeting the vlPFC and its key functions (e.g. cognitive control, response inhibition) could have utility in the treatment of pathological gambling. Clinicians may also adapt standard interventions to buffer the impact of ventrolateral dysfunction on real-life functioning (i.e. instructing clients to pay attention to negative feedback and training them to generate and rehearse alternative strategies).

Our study has several strengths, including the direct comparison of cocaine and gambling groups with minimum exposure to alcohol/other drugs and without the confounding effects of psychiatric comorbidities. Moreover, recruitment was based on consecutive admissions to public treatment centres, hence making the sample truly clinically representative. Further, both groups were carefully supervised for continuous abstinence during the study, thus ruling out confounding effects of acute drug use/gambling, withdrawal or craving. Current drug use was objectively monitored throughout study completion using ongoing urine toxicologies that ruled out any use of cocaine and other drugs. There is however a potential limitation in the supervision of gambling abstinence in the gambling group, which was based on reports from both participants and significant others, but is still susceptible to reporting biases. ${ }^{54,55}$ Moreover, the genetic analyses in particular should be interpreted in the context of the relatively small sample size and the examination of a single cognitive domain and, as such, should be appraised as preliminary. Future studies are warranted to examine the additive contribution of different polymorphisms across the dopamine genetic pathway, and the clinical relevance of this reversal learning-related neuroimaging phenotype to cocaine and gambling treatment outcomes.

\begin{abstract}
Antonio Verdejo-Garcia, PhD, School of Psychology and Psychiatry, Monash University, Melbourne, Australia, Institute of Neuroscience F. Oloriz, Universidad de Granada, Granada, Spain and Red de Trastornos Adictivos, Universidad de Granada. Granada, Spain; Luke Clark, PhD, Department of Psychology, Centre for Gambling Research at UBC, University of British Columbia, Canada; Juan Verdejo-Román, MSc, Institute of Neuroscience F. Oloriz, Universidad de Granada, Granada, Spain; Natalia Albein-Urios, PhD, Institute of Neuroscience F. Oloriz, Universidad de Granada, Granada, Spain; José M. Martinez-Gonzalez, PhD, Red de Trastornos Adictivos, Universidad de Granada, Granada, Spain and Centro Provincial de Drogodependencias, Diputacion de Granada, Granada, Spain; Blanca Gutierrez, Drogodependencias, Diputacion de Granada, Granada, Spain; Blanca Gutierrez,
PhD, Institute of Neuroscience F. Oloriz, Universidad de Granada, Granada, Spain, Department of Psychiatry, Universidad de Granada, Granada, Spain and CIBERSAM, Carlos III Health Institute, Barcelona, Spain; Carles Soriano-Mas, PhD, Department of Psychiatry, Bellvitge Biomedical Research Institute-IDIBELL, Barcelona, Spain and CIBERSAM, Carlos III Health Institute, Barcelona, Spain, Department of Psychobiology and Methodology of Health Sciences, Universitat Autònoma de Barcelona, Spain
\end{abstract}

Correspondence: Antonio Verdejo-Garcia, School of Psychology and Psychiatry, Monash University, 3800 Wellington Rd. Clayton Campus, Melbourne, Australia. Email address: Antonio.Verdejo@monash.edu

First received 1 Jun 2014, final revision 18 Nov 2014, accepted 23 Nov 2014

\section{Funding}

This study has been funded by grants from the Spanish Ministry of Health; project grant COPERNICO, Drug Abuse Plan (Plan Nacional sobre Drogas Convocatoria 2009) and program grant RETICS, Carlos III Health Institute (Instituto de Salud Carlos III, Red de Trastornos Adictivos). CS-M is funded by a 'Miguel Servet' contract from the Carlos III Health Institute (CP10/00604)

\section{References}

1 American Psychiatric Association. Diagnostic and Statistical Manual of Mental Disorders, 5th ed. American Psychiatric, 2013.

2 Lucantonio F, Stalnaker TA, Shaham Y, Niv Y, Schoenbaum G. The impact of orbitofrontal dysfunction on cocaine addiction. Nat Neurosci 2012; 15: $358-66$.

3 Turner TH, LaRowe S, Horner MD, Herron J, Malcolm R. Measures of cognitive functioning as predictors of treatment outcome for cocaine dependence. J Subst Abuse Treat 2009; 37: 328-34.

4 Clark L, Cools R, Robbins TW. The neuropsychology of ventral prefrontal cortex: decision-making and reversal learning. Brain Cogn 2004; 55: 41-53.

5 Izquierdo A, Jentsch JD. Reversal learning as a measure of impulsive and compulsive behavior in addictions. Psychopharmacology 2012; 219: 607-20.

6 Leeman RF, Potenza MN. Similarities and differences between pathological gambling and substance use disorders: a focus on impulsivity and compulsivity. Psychopharmacology 2012; 219: 469-90.

7 Fernández-Serrano MJ, Perales JC, Moreno-López L, Pérez-García M, Verdejo-García A. Neuropsychological profiling of impulsivity and compulsivity in cocaine dependent individuals. Psychopharmacology 2012; 219: 673-83.

8 Ersche KD, Roiser JP, Robbins TW, Sahakian BJ. Chronic cocaine but not chronic amphetamine use is associated with perseverative responding in humans. Psychopharmacology 2008; 197: 421-31.

9 Vonmoos M, Hulka LM, Preller KH, Jenni D, Baumgartner MR, Stohler R, et al. Cognitive dysfunctions in recreational and dependent cocaine users: role of attention-deficit hyperactivity disorder, craving and early age at onset. Br J Psychiatry 2013; 203: 35-43.

10 Vanes LD, van Holst RJ, Jansen JM, van den Brink W, Oosterlaan J, Goudriaan AE. Contingency learning in alcohol dependence and pathological gambling: learning and unlearning reward contingencies. Alcohol Clin Exp Res 2014 (doi:10.1111/acer.12393).

11 Cools R, Clark L, Owen, AM, Robbins TW. Defining the neural mechanisms of probabilistic reversal learning using event-related functional magnetic resonance imaging. J Neurosci 2002; 22: 4563-7. 
12 Camchong J, MacDonald III AV, Nelson B, Bell C, Mueller BA, Specker S, et al Frontal hyperconnecivity related to discounting and reversal learning in cocaine subjects. Biol Psychiatry 2011; 69: 1117-23.

13 Ersche KD, Roiser JP, Abbott S, Craig KJ, Muller U, Suckling J, et al. Response perseveration in stimulant dependence is associated with striatal dysfunction and can be ameliorated by a $\mathrm{D}(2 / 3)$ receptor agonist. Biol Psychiatry 2011; 70: 754-62.

14 de Ruiter MB, Veltman DJ, Goudriaan AE, Oosterlaan J, Sjoerds Z, van den Brink W. Response perseveration and ventral prefrontal sensitivity to reward and punishment in male problem gamblers and smokers. Neuropsychopharmacology 2009; 34: 1027-38.

15 Jocham G, Klein TA, Neumann J, von Cramon DY, Reuter M, Ullsperger M. Dopamine DRD2 polymorphism alters reversal learning and associated neura activity. J Neurosci 2009; 29: 3695-704.

16 Noble EP. Addiction and its reward process through polymorphisms of the D2 dopamine receptor gene: a review. Eur Psychiatry 2000; 15: 79-89.

17 Madhavan A, Argilli E, Bonci A, Whistler JL. Loss of D2 dopamine receptor function modulates cocaine-induced glutamatergic synaptic potentiation in the ventral tegmental area. J Neurosci 2013; 33: 12329-36.

18 Volkow ND, Fowler JS, Wang GJ, Hitzemann R, Logan J, Schlyer DJ, et al. Decreased dopamine D2 receptor availability is associated with reduced frontal metabolism in cocaine abusers. Synapse 1993; 14: 169-77.

19 Volkow ND, Chang L, Wang GL, Fowler JS, Ding YS, Sedler M, et al. Low leve of brain dopamine D2 receptors in methamphetamine abusers: association with metabolism in the orbitofrontal cortex. Am J Psychiatry 2011; 158: 2015-21.

20 Porter JN, Olsen AS, Gurnsey K, Dugan BP, Jedema HP, Bradberry CW. Chronic cocaine self-administration in rhesus monkeys: impact on associative learning, cognitive control, and working memory. J Neurosci 2011; 31: 4926-34

21 Lee B, Groman S, London ED, Jentsch JD. Dopamine D2/D3 receptors play a specific role in the reversal of a learned visual discrimination in monkeys. Neuropsychopharmacology 2007; 32: 2125-34.

22 Fernandez-Serrano MJ, Perez-Garcia M, Schmidt Rio-Valle J, Verdejo-Garcia A. Neuropsychological consequences of alcohol and drug abuse on different components of executive functions. J Psychopharmacol 2010; 24: 1317-32.

23 Woicik PA, Urban C, Alia-Klein N, Henry A, Maloney T, Telang F, et al. A pattern of perseveration in cocaine addiction may reveal neurocognitive processes implicit in the Wisconsin Card Sorting Test. Neuropsychologia 2011; 49: 1660-9.

24 van Holst RJ, de Ruiter MB, van den Brink W, Veltman DJ, Goudriaan AE. A voxel-based morphometry study comparing problem gamblers, alcohol abusers, and healthy controls. Drug Alcohol Depend 2012; 124: 142-8.

25 Clark L, Limbrick-Oldfield EH. Disordered gambling: a behavioral addiction. Curr Opin Neurobiol 2013; 23: 655-9.

26 Limbrick-Oldfield EH, van Holst RJ, Clark L. Fronto-striatal dysregulation in drug addiction and pathological gambling: consistent inconsistencies? Neurolmage Clin 2013; 2: 385-93.

27 Kaufman JC, Kaufman AS. Time for the changing of the guard: a farewell to short forms of intelligence tests. J Psychoeducational Assess 2001; 19: 245-67.

28 Verdejo-Garcia AJ, Lopez-Torrecillas F, Aguilar de Arcos F, Perez-Garcia M. Differential effects of MDMA, cocaine, and cannabis use severity on distinctive components of the executive functions in polysubstance users: a multiple regression analysis. Addict Behav 2005; 30: 89-101.

29 Lobo A, Perez-Echeverria MJ, Artal J. Validity of the scaled version of the General Health Questionnaire (GHQ-28) in a Spanish population. Psychol Med 1986; 16: 135-40.

30 López-Castromán J, Vaquero-Lorenzo C, Perez-Rodriguez MM, Diaz-Hernandez M, Fernandez-Piqueras J, Saiz-Ruiz J, et al. Gender effect on association between DRD2 polymorphism and substance dependence in a Spanish sample. Drug Alcohol Depend 2009; 101: 210-2.

31 Perez de los Cobos J, Baiget M, Trujols J, Sinol N, Volpini V, Banuls E, et al Allelic and genotypic associations of DRD2 Taql A polymorphism with heroin dependence in Spanish subjects: a case control study. Behav Brain Funct 2007; 3: 25 .

32 First MB, Spitzer RL, Gibbon M, Williams JBW. Structured Clinical Interview for DSM-IV Axis I Disorders (SCID-I). American Psychiatric Press, 1997.

33 Loranger AW, Sartorius N, Andreoli A, Berger P, Buchheim P, Channabasavanna SM, et al. The International Personality Disorder Examination. The World Health Organization/Alcohol, Drug Abuse, and
Mental Health Administration international pilot study of personality disorders. Arch Gen Psychiatry 1994; 51: 215-24.

34 Conners CK. Clinical use of rating scales in diagnosis and treatment of attention-deficit/hyperactivity disorder. Pediatr Clin North Am 1999; 46 $857-70$.

35 Ritchie T, Noble EP. Association of seven polymorphisms of the D2 dopamine receptor gene with brain receptor-binding characteristics. Neurochem Res 2003; 28: 73-82.

36 Ward BD. Alphasim Program Documentation for AFNI, Simultaneous Inference for fMRI Data. Medical College of Wisconsin, Milwaukee. 2000 (http://afni.nimh.nih.gov/pub/dist/doc/manual/AlphaSim.pdf).

37 Song XW, Dong ZY, Long XY, Li SF, Zuo XN, Zhu CZ, et al. REST: a toolkit for resting-state functional magnetic resonance imaging data processing. PLOS ONE 2011; 6: e25031.

38 Mitchell DG, Luo Q, Avny SB, Kasprzycki T, Gupta K, Chen G, et al. Adapting to dynamic stimulus-response values: differential contributions of inferior frontal, dorsomedial, and dorsolateral regions of prefrontal cortex to decision making. J Neurosci 2009; 29: 10827-34.

39 Tabibnia G, Monterosso JR, Baicy K, Aron AR, Poldrack RA, Chakrapani S, et al. Different forms of self-control share a neurocognitive substrate. J Neurosci 2011; 31: 4805-10.

40 Glaser YG, Zubieta JK, Hsu DT, Villafuerte S, Mickey BJ, Trucco EM, et al. Indirect effect of corticotropin-releasing hormone receptor 1 gene variation on negative emotionality and alcohol use via right ventrolateral prefrontal cortex. J Neurosci 2014; 34: 4099-107.

41 Smith DG, Jones PS, Bullmore ET, Robbins TW, Ersche KD. Cognitive control dysfunction and abnormal frontal cortex activation in stimulant drug users and their biological siblings. Transl Psychiatry 2013; 3: e257.

42 Kroener S, Chandler L, Phillips PE, Seamans JK. Dopamine modulates persistent synaptic activity and enhances the signal-to-noise ratio in the prefrontal cortex. PLOS ONE 2009; 4: e6507.

43 Cohen MX, Krohn-Grimberghe A, Elger CE, Weber B. Dopamine gene predicts the brain's response to dopaminergic drug. Eur J Neurosci 2007; 26 3652-60.

44 Ashare RL, Wileyto EP, Ruparel K, Goelz PM, Hopson RD, Valdez JN, et al. Effects of tolcapone on working memory and brain activity in abstinent smokers: a proof-of-concept study. Drug Alcohol Depend 2013; 133: 852-6.

45 Clark CR, Egan GF, McFarlane AC, Morris P, weber D, Sonkkilla C, et al. Updating working memory for words: a PET activation study. Hum Brain Mapp 2000; 9: 42-54.

46 Wager TD, Jonides J, Reading S. Neuroimaging studies of shifting attention: a meta-analysis. Neurolmage 2004; 22: 1679-93.

47 Hearing $M$, Kotecki L, Marron Fernandez de Velasco E, Fajardo-Serrano A, Chung HJ, Lujan R, et al. Repeated cocaine weakens GABA(B)-Girk signaling in layer 5/6 pyramidal neurons in the prelimbic cortex. Neuron 2013; 80 : 159-70.

48 Kasanetz F, Lafourcade M, Deroche-Gamonet V, Revest JM, Berson N, Balado $\mathrm{E}$, et al. Prefrontal synaptic markers of cocaine addiction-like behavior in rats. Mol Psychiatry 2013; 18: 729-37.

49 Mihindou C, Guillem K, Navailles S, Vouillac C, Ahmed SH. Discriminative inhibitory control of cocaine seeking involves the prelimbic prefrontal cortex. Biol Psychiatry 2013; 73: 271-9.

50 Barros-Loscertales A, Garavan H, Bustamante JC, Ventura-Campos N, Llopis JJ, Belloch V, et al. Reduced striatal volume in cocaine-dependent patients. Neuroimage 2011; 56: 1021-6.

51 Dombrovski AY, Clark L, Siegle GJ, Butters MA, Ichikawa N, Sahakian BJ et al. Reward/punishment reversal learning in older suicide attempters. Am J Psychiatry 2010; 167: 699-707.

52 Albein-Urios N, Martinez-González JM, Lozano O, Clark L, Verdejo-García A. Comparison of impulsivity and working memory in cocaine addiction and pathological gambling: implications for cocaine-induced neurotoxicity. Drug Alcohol Depend 2012; 126: 1-6.

53 Wesley MJ, Bickel WK. Remember the future II: meta-analyses and functional overlap of working memory and delay discounting. Biol Psychiatry 2014; 75 : 435-48. doi:10.1016/j.biopsych.2013.08.008.

54 Kuentzel JG, Henderson MJ, Melville CL. The impact of social desirability biases on self-report among college student and problem gamblers. J Gambl Stud 2008; 24: 307-19.

55 Toce-Gerstein M, Gerstein DR, Volberg RA. A hierarchy of gambling disorders in the community. Addiction 2003; 98: 1661-72. 